\title{
Tractography-Based Parcellation of the Cortex Using a Spatially-Informed Dimension Reduction of the Connectivity Matrix
}

\author{
Pauline Roca ${ }^{1,2}$, Denis Rivière ${ }^{1,2}$, Pamela Guevara ${ }^{1,2}$, Cyril Poupon ${ }^{1,2}$, \\ and Jean-François Mangin ${ }^{1,2}$ \\ 1 CEA Saclay, Neurospin/LNAO, Bât 145, 91191 Gif-sur-Yvette cedex, France \\ 2 Institut Fédératif de Recherche 49, Gif-sur-Yvette, France
}

\begin{abstract}
Determining cortical functional areas is an important goal for neurosciences and clinical neurosurgery. This paper presents a method for connectivity-based parcellation of the entire human cortical surface, exploiting the idea that each cortex region has a specific connection profile. The connectivity matrix of the cortex is computed using analytical Q-ball-based tractography. The parcellation is achieved independently for each subject and applied to the subset of the cortical surface endowed with enough connections to estimate safely a connectivity profile, namely the top of the cortical gyri. The key point of the method lies in a twofold reduction of the connectivity matrix dimension. First, parcellation amounts to iterating the clustering of Voronoï patches of the cortical surface into parcels endowed with homogeneous profiles. The parcels without intersection with the patch boundaries are selected for the final parcellation. Before clustering a patch, the complete profiles are collapsed into short profiles indicating connectivity with a set of putative cortical areas. These areas are supposed to correspond to the catchment basins of the watershed of the density of connection to the patch computed on the cortical surface. The results obtained for several brains are compared visually using a coordinate system.
\end{abstract}

\section{Introduction}

Characterization of functional areas and their relationships is a key to understand how brain works. Recent studies follow this goal and represent the brain as a graph, analyzing this network using graph theory [1/2 3. The construction of this human "connectome" requires the definition of basic structural elements (nodes of the network), which can be chosen at different scales: level of single neurons (microscale), neuronal populations (mesoscale), or anatomically distinct brain regions (macroscale) 4]. In this context, a parcellation of the human brain into functional areas can be a solution to the definition of a first network at a macroscale. Following the idea that each brain module has a specific connectional fingerprint [5], the connectivity information based on diffusion-weighted imaging is an interesting candidate for defining the connectome nodes. A comparison with a functional connectivity study could be very useful for a validation 67. 
The main trouble to deal with when addressing connectivity-based parcellations from diffusion imaging is the huge dimension of the raw connectivity profiles. For instance, a standard mesh of the cortical surface is made up of more than 10000 nodes. Segmenting this surface into parcels with homogeneous connectivity profiles amounts to performing clustering among vectors of dimension 10 000. Clustering is prone to the well-known curse of dimensionality. Therefore, reduction dimension seems mandatory. Several approaches have been proposed for this purpose. The first one is based on a segmentation of the brain to collapse the connectivity profiles: all the tracts reaching the same segment are summed up. This segmentation can be anatomical, for instance based on lobes or gyri 8 8 10, but the same idea could be applied with fMRI-based activation maps. In the papers cited above, the segmentation used for collapsing was based on individual data. This segmentation could also stem from the results of voxelbased group analysis or the results of invasive anatomical tracing performed in primates [11]. Such a priori knowledge is mapped into individual space using spatial normalization or sulcal landmarks. Finally, performing the clustering in a small area of the brain can overcome the curse of dimensionality. It was shown that Broca's area 1213] and lateral premotor cortex [14 can be reproducibly parcellated from connectivity profiles towards the whole brain.

In this paper, the goal is to design a dimension reduction strategy efficient enough to allow reproducible parcellation of the whole cortical surface. We explore the possibility to reach this goal without using any information about the geometry of the cortical surface (sulco-gyral anatomy, coordinate systems). This is a challenging objective, but in our opinion, it has to be tackled to examine the extent to which one can get rid of hypotheses about the connectome structure that could bias the connectivity-based parcellation. The objective is all the more challenging since the parcellation is applied independently on each subject. Hence tractography errors cannot be averaged out by a group analysis. Our method is based on three key points that are described in the paper:

1. The algorithm is applied to the subset of the cortical surface endowed with enough connections to safely estimate a connectivity profile. This subset turns out to match the top of the cortical gyri. In this paper, we focus on long diffusion-based tracts that are supposed to convey more reproducible connectivity information than short U-fiber tracts.

2. The clustering is not performed on a whole brain basis but with an iterative patch by patch strategy.

3. The connectivity profiles are collapsed in an adaptive way for each patch to be parcellated: the segmentation for collapsing is based on the catchment basins of the watershed of the density of connection to the patch computed on the cortical surface.

An original visualization of the resulting parcellation has been developed. It consists in superimposing on each parcel a small replica of the whole brain indicating either the density of connection to the underlying parcel or the tracts terminating in this parcel. We compare the results obtained with four subjects using a coordinate system provided by BrainVISA based on the main sulci [15]. 


\section{Materials and Method}

\subsection{Data and Pre-processings}

The present study has been performed on four subjects of the NMR public database [16]. Diffusion weighted data were acquired with HARDI scheme, resulting in high-quality datasets based on 200 directions and a $b$ value of $3000 \mathrm{~s} / \mathrm{mm}^{2}$. Perfect alignment between diffusion data and a T1-weighted $1 \mathrm{~mm}$ resolution image is achieved using a dedicated set of distortion corrections. The T1 pipeline of BrainVISA open software1 is used to compute a mesh $S$ of the cortical surface and a sulcus-based coordinate system used to compare subjects. The mesh is made up of two hemispheric spherical representations of the grey/white interface including about 40000 vertices. The direct parcellation of the cortical surface capitalizes on the current knowledge of the columnar organization of the cortex: local populations of neurons form elementary processing units organized orthogonally to the cortex surface [4].

A deterministic tractography based on analytical Q-ball field [17] is performed using BrainVISA diffusion toolbox. The tracts are the trajectories of particles with inertia, which is leading to regularize curvature [18. Tractography was initiated from two seeds in each voxel of the high resolution white matter mask computed from the T1-weighted acquisition, in both retrograde and orthograde directions, according to the maximal direction of the underlying ODF. Tracking is stopped either when the particle exits the propagation mask, when the angle between the two last moves exceeds $30^{\circ}$, or when the tract length exceeds 200 $\mathrm{mm}$. Finally, tracts shorter than $20 \mathrm{~mm}$ were filtered out, leading to a set of about 1.5 millions tracts per subject.

Then histograms of tract lengths were computed for each subject (see Fig. 1) showing a reproducible two mode distribution. This study explores the connectivity information provided by the second mode defined by a $10 \mathrm{~cm}$ threshold. This ad hoc choice is based on the hypothesis that long range bundles are more reproducible across individuals than shorter U-fiber bundles. This threshold might at first glance look very high, but histograms show that about one third of the tracts are longer than $10 \mathrm{~cm}$.

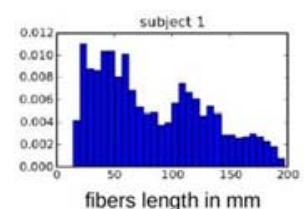

fibers length in $\mathrm{mm}$

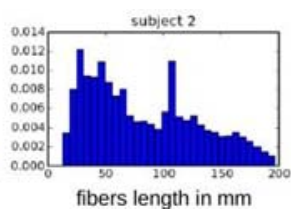

fibers length in $\mathrm{mm}$

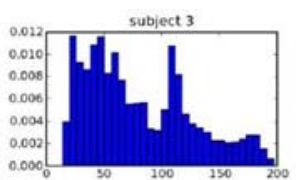

fibers length in $\mathrm{mm}$

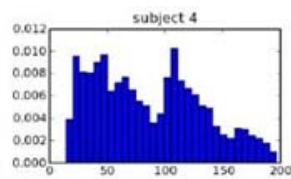

fibers length in $\mathrm{mm}$

Fig. 1. Fibers length histograms

${ }^{1}$ http://brainvisa.info 


\subsection{Cortical Connection Matrix Construction}

The cortex surface is divided into Voronoi cells centered around the surface mesh vertices. Then a raw connectivity matrix $C^{\text {raw }}$ is computed by counting fibers connecting cells [19]. The raw matrix $C^{\text {raw }}$ is then smoothed over the surface to account for a reasonable uncertainty on the tracking result. The smoothed matrix $C$ used further results from the convolution of $C^{\text {raw }}$ with a Gaussian. Gaussian weigths stem from geodesic distances computed using fast marching over triangulations [20. The Gaussian standard deviation has been set to $5 \mathrm{~mm}$.

\subsection{Parcellation of the Cortex Mesh}

The cortex surface $S$ is parcellated according to an iterative algorithm summarized in Fig 2. The algorithm is applied to the subset of the cortical surface endowed with enough connections to safely estimate a connectivity profile. The density of connexions for each vertice is the $L_{1}$-norm of the corresponding row in $C$. A threshold on density provides the initial domain which turns out to correspond to the top of the gyri. In the following, we call resp. cortex sub-mesh connected component of the graph formed by the evoluting cortex triangulation S, patch Voronoï parcellation's segment, parcel final parcellation's component.

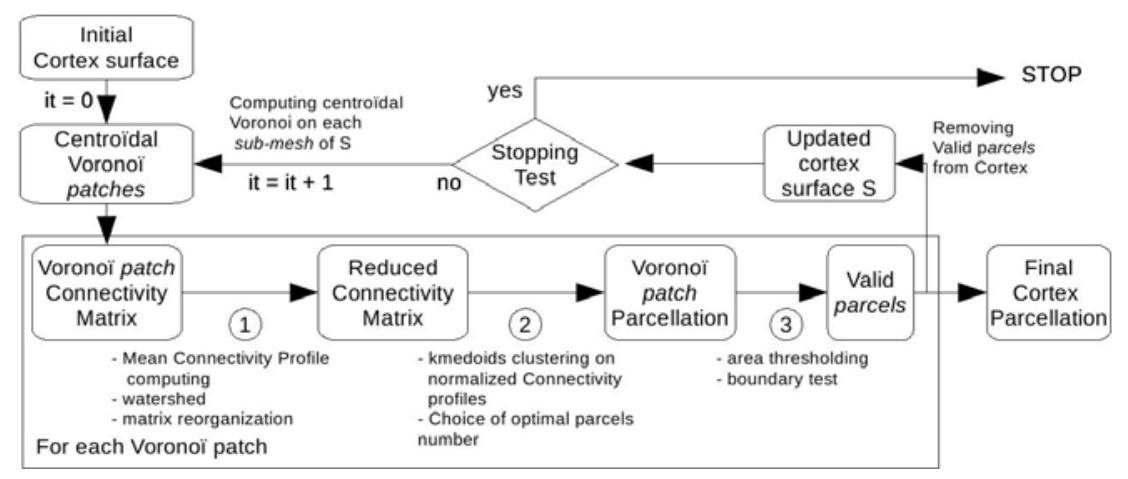

Fig. 2. A sketch of the parcellation Method

Each iteration begins with the random tessalation of the current domain into large patches of similar sizes. This splitting of the domain, aiming at reducing the complexity of the clustering problem, is performed cortex sub-mesh by cortex sub-mesh. Each cortex sub-mesh is split using the Centroïdal Voronoï tessalation algorithm [21]: a set of points is chosen randomly in the domain, a first Voronoï diagram is computed from these points, then the process is iterated using the Voronoï patch centroïds as seeds. We stop the process after five iterations. Assuming a value of 150 areas per hemisphere, each individual cortical area would occupy an average of $6 \mathrm{~cm}^{2}$, with some much larger areas and some 


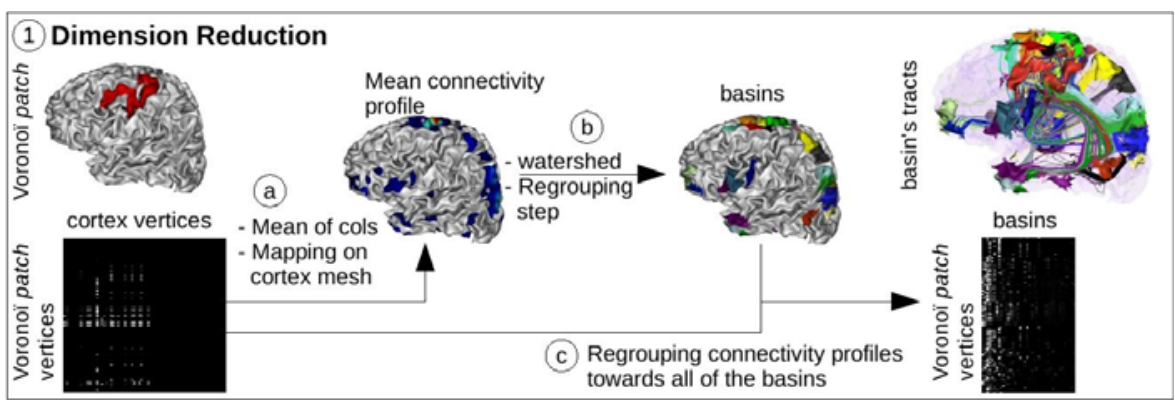

Fig. 3. Dimension Reduction Step 1. In the composite image on the right, the tract colors correspond to the color of the basin they connect with the Voronoi patch.

much smaller [22]. The number of random points used aims to split the domain into patches whose area correspond to ten times this average area of $6 \mathrm{~cm}^{2}$.

Then each Voronoï patch is segmented into parcels with homogeneous connectivity profiles. The parcels larger than a minimum area and which do not intersect with the patch boundary are added to the final parcellation and removed from the domain during the next iterations. The algorithm stops as soon as one iteration did not add any parcel in the final result. The minimum area of a parcel is set to $2 \mathrm{~cm}^{2}$, to account for the size variability of functional areas.

The clustering of the profiles of a Voronoï patch begins with an adaptive dimension reduction (Fig (3). The mean connectivity profile of the patch is computed and represented as a texture on the complete cortical surface. This texture is the density of connection to this patch (Fig[3.a). Then, a watershed is computed for this texture in order to split the cortical surface into catchment basins. The set of basins is pruned using a standard merging procedure based on depth and area. Each basin catches a set of tracts supposed to connect the patch with a meaningful brain area. The smallest basins are discarded in order to keep only $95 \%$ of the tract density (Fig 3 , b) and the connectivity profiles are collapsed using the resulting set of basins (Fig $3 \mathrm{c}$ ). Finally, the reduced connectivity profiles are normalized $\left(L_{2}\right.$-norm) and clustered using a kmedoids algorithm (PAM in R language [23]). The number of clusters $K$ which gives the highest average silhouette width is considered as optimal [23].

\section{$3 \quad$ Results}

The method finds on average 140 parcels per hemiphere. The complexity of the data manipulated in this paper and the absence of gold standard prevents a straightforward evaluation of the results obtained for the four subjects. Hence we have decided to provide original visualizations that convey some insights about the level of reproducibility. A 3D object gathering all connectivity profiles is created. The parcellations are projected to an inflated cortex surface. For each parcel, a small cortex mesh textured with the normalized mean connectivity 

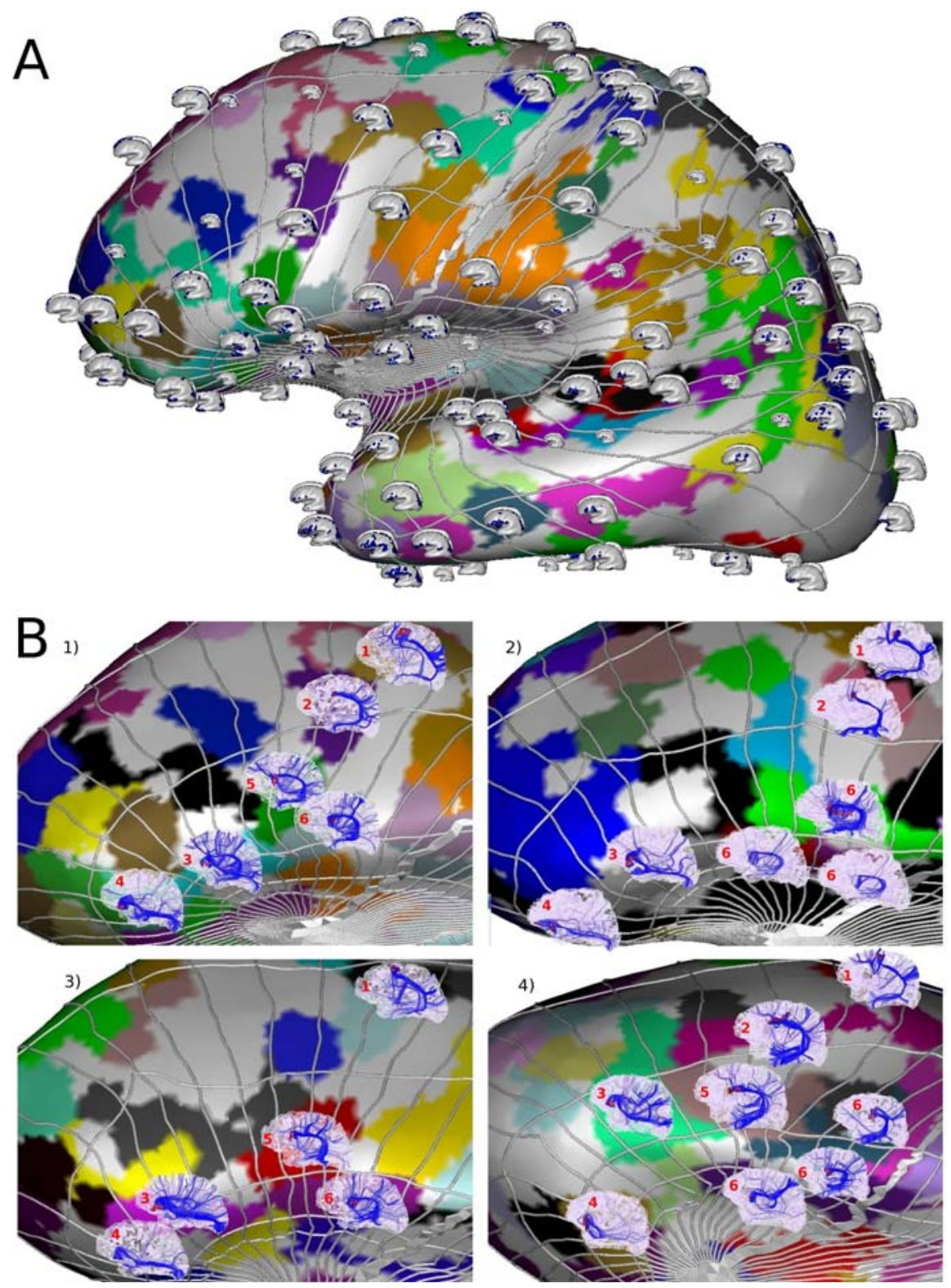

Fig. 4. A The parcels and their connectivity profiles for subject 1 . The grid is a spherical coordinate system based on sulci. The thick line is the central sulcus. B Zoom on Parcellation around Broca's Area for the four subjects. A tract image is used to describe the profiles that show similarities across subjects. 
profile of the parcel is put in the parcel's centroïd. An additional structure representing meridians and parallels of a sulcus-based spherical coordinate system is superimposed [15] (see Fig 4, A).

While the coordinate system could allow the definition of quantitative evaluations of the reproducibility of the parcellation, we prefer to rely on visualization. The localization power of sulci relative to fiber bundles, is indeed a subject of research. The $3 \mathrm{D}$ scenes of profiles can be rotated in $3 \mathrm{D}$ in order to explore similarities across subjects. Additional windows can be opened to visualize the tracts: clicking on a parcel selects the connected tracts. We use this second functionality to create composite images of the 4 subjects centered around Broca's area. 3D images of the tracts were superimposed on the parcels whose profiles could be visually matched across subjects (see Fig $4 \mathbf{B}$ ). Tract images provide more information than profile images.

\section{Discussion}

Achieving a parcellation of the human brain cortex is fraught with difficulties, especially in the context of this study. A connectivity-based parcellation without anatomical a priori and performed independently for each subject is very ambitious. This study is still largely exploratory, relying on several parameters such as tracking method, fiber length threshold, clustering algorithm or optimal cluster's number, whose influence should be studied. Although this paper does not reach clearly reproducible results, the original visualization presented in this work leads to a better understanding of connectivity data and could also be used for studies of functional connectivity or any other vertex-based correlation measure.

Diffusion-based tractography is a rapidly developing field. The new generation of $1 \mathrm{~mm}$ resolution acquisition that can now be achieved with highly parallel imaging and high fields could qualitatively change the individual parcellation obtained by our method. Furthermore, using the most advanced ODF deconvolution tools or probabilistic tracking could also improve our results. Nevertheless, the lack of reproducibility highlighted in this paper will lead us to address the parcellation at the level of the group of subjects. Surface-based coordinate system can be used to design such group studies. Other improvements will stem from using a segmentation of the deep structures (thalamus, basal ganglia, etc.) to increase the information coded in the connectivity profiles. Finally, a threshold free approach will be interesting for tuning the contributions of each tract to the profile according to its length.

\section{References}

1. Bullmore, E., Sporns, O.: Complex brain networks: graph theoretical analysis of structural and functional systems. Nature Neuroscience 10 (2009)

2. Iturria-Medina, Y., Canales-Rodriguez, E., Melie-Garcia, L.: Characterizing brain anatomical connections using diffusion weighted mri and graph theory. NeuroImage 36, 645-660 (2007) 
3. Hagmann, P., Cammoun, L., Gigandet, X., et al.: Mapping the structural core of human cerebral cortex. PLOS Computational Biology 6(7), 1479-1493 (2008)

4. Sporns, O., Tononi, G., Kötter, R.: The human connectome: A structural description of the human brain. PLOS Comp. Biology 1(4), 245-251 (2005)

5. Passingham, R., Stephan, K., Kötter, R.: The anatomical basis of functional localization in the cortex. Nature 3, 606-616 (2002)

6. Honey, C.J., Sporns, O., et al.: Predicting human resting-state functional connectivity from structural connectivity. Proc. Natl. Acad. Sci. 106(6), 2035-2040 (2009)

7. Skudlarski, P., Jagannathan, K., Calhoun, V., et al.: Measuring brain connectivity: Diffusion tensor imaging validates resting state temporal correlations. NeuroImage $43(3), 554-561(2008)$

8. Behrens, T., Johansen-Berg, H., Woolrich, M.W., et al.: Non-invasive mapping of connections between human thalamus and cortex using diffusion imaging. Nature Neuroscience 6(7), 750-757 (2003)

9. Perrin, M., Cointepas, Y., Cachia, A.: Connectivity-based parcellation of the cortical mantle using q-ball diffusion imaging. Int. J. Biomed. Imaging (2008)

10. Guevara, P., Perrin, M., Cathier, P.: et al.: Connectivity-based parcellation of the cortical surface using Q-ball imaging. In: 5th Proc. IEEE ISBI, Paris, France, pp. 903-906 (2008)

11. Rushworth, M., Behrens, T., Johansen-Berg, H.: Connections patterns distinguish 3 regions of human parietal cortex. Cerebral Cortex 16, 1418-1430 (2005)

12. Anwander, A., Tittgemeyer, M., von Cramon, D., et al.: Connectivity-based parcellation of brocas area. Cerebral Cortex 17, 816-825 (2007)

13. Klein, J., Behrens, T., Robson, M., et al.: Connectivity-based parcellation of human cortex using diffusion mri: Establishing reproducibility, validity and observer independence in ba 44/45 and sma/pre-sma. NeuroImage 34, 204-211 (2007)

14. Tomassini, V.: Relating connectional architecture to grey matter function in the human lateral premotor cortex using functional and diffusion imaging. HBM (2008)

15. Clouchoux, C., Coulon, O., Rivière, D.: Anatomically constrained surface parameterization for cortical localization. In: Duncan, J.S., Gerig, G. (eds.) MICCAI 2005. LNCS, vol. 3750, pp. 344-351. Springer, Heidelberg (2005)

16. Poupon, C., Poupon, F., Allirol, L.: NMR: a free database dedicated to the anatomofunctional study of the human brain connectivity. In: HBM (2006)

17. Descoteaux, M., Deriche, R., Knösche, T.R., et al.: Deterministic and probabilistic tractography based on complex fibre orientation distributions. IEEE Transactions on Medical Imaging 28, 269-286 (2009)

18. Perrin, M., Poupon, C., Cointepas, Y.: Fiber tracking in q-ball fields using regularized particle trajectories. Inf. Process Med. Imaging 19, 52-63 (2005)

19. Cathier, P., Mangin, J.-F.: Registration of cortical connectivity matrices. In: Proc. MMBIA 2006, New York, USA (2006)

20. Kimmel, R., Sethian, J.A.: Computing geodesic paths on manifolds. Proc. Natl. Acad. Sci. 95, 8431-8435 (1998)

21. Du, Q., Faber, V., Gunzburger, M.: Centroidal voronoi tessellations: Applications and algorithms. Society for Industrial and Applied Mathematics Review 41(4), 637-676 (1999)

22. Van Essen, D.C., Dierker, D.L.: Surface-based and probabilistic atlases of primate cerebral cortex. Neuron 56, 209-225 (2007)

23. Kaufmann, L., Rousseeuw, P.: Finding groups in data: an introduction to cluster analysis. Wiley Interscience, Hoboken (1990) 